\title{
Mechanical topological insulator in zero dimensions
}

\author{
Natalia Lera and J. V. Alvarez \\ Departamento de Física de la Materia Condensada, Universidad Autónoma de Madrid, Madrid 28049, Spain \\ and Spain Condensed Matter Physics Center (IFIMAC) and Instituto Nicolás Cabrera, \\ Universidad Autónoma de Madrid, Madrid 28049, Spain
}

(Received 1 March 2018; published 30 April 2018)

\begin{abstract}
We study linear vibrational modes in finite isostatic Maxwell lattices, mechanical systems where the number of degrees of freedom matches the number of constraints. Recent progress in topological mechanics exploits the nontrivial topology of BDI class Hamiltonians in one dimenson and arising topological floppy modes at the edges. A finite frame, or zero-dimensional system, also exhibits a nonzero topological index according to the classification table. We construct mechanical insulating models in zero dimensions that complete the BDI classification in the available real space dimensions. We compute and interpret its nontrivial invariant $\mathbb{Z}_{2}$.
\end{abstract}

DOI: 10.1103/PhysRevB.97.134118

\section{INTRODUCTION}

Zero-dimensional Hamiltonians describe systems with a finite number of energy levels. The classification table for insulators and superconductors [1-3] shows that there are five topological classes with nontrivial topology in zero dimensions. This means there is not a continuous transformation between any two Hamiltonians in each class. Three out of these five classes with nontrivial topology are A, AI, and AII classes, whose topological invariant in zero dimensions is the number of occupied levels. Two systems of the same dimension will be topologically equivalent if they have the same number of filled levels, otherwise an interpolation between the two must have a zero level or a gap closing. For superconducting quantum dots, level crossing in the D class [4] is protected by fermion parity [5-7]. For the BDI class, we show the crossings are protected by sublattice symmetry.

The existence of a nontrivial one-dimensional (1D) topological invariant in the BDI class has been exploited to study isostatic Maxwell lattices. Topology plays an important role in these elastic systems, giving rise to topologically protected phonon-edge modes at zero frequency (or floppy modes). Maxwell lattices appear in nature in systems such as fiber networks [8] and jammed packings [9], as well as in artificial metamaterials as mass-spring systems (including other mechanical devices), both insulators and Weyl semimetals [10-21].

Isostatic lattices have the same number of constraints as degrees of freedom; therefore we may say they are at the verge of mechanical instability. The equilibrium matrix, $Q$, is a square matrix and physically meaningful $[10,11]$. $Q$ relates tensions of springs in mass-spring models with forces in each degree of freedom. Its transpose $C=Q^{t}$, the compatibility matrix (also called the rigidity matrix), relates the displacement of the degrees of freedom with the springs' elongation. We can define a Hamiltonian which contains more information than the dynamical matrix, $D$, of the elastic system as follows:

$$
H_{C}=\left(\begin{array}{cc}
0 & C \\
C^{t} & 0
\end{array}\right)
$$

The square of the Hamiltonian is as follows:

$$
H_{C}^{2}=\left(\begin{array}{cc}
\tilde{D} & 0 \\
0 & D
\end{array}\right)
$$

where $\tilde{D}=C C^{t}$ is the dual partner of $D=C^{t} C$. The topological information is lost in the dynamical matrix, a regular method for studying elastic systems. We need to turn to a compatibility matrix or an equilibrium matrix to consider the topology of the systems and the propagation of protected edge modes. The construction of $H_{C}$ guarantees chiral symmetry. Moreover, the reality condition in real space of the compatibility matrix, $C(k)=C^{*}(-k)$, imposes the time-reversal symmetry squaring to 1 . Or in zero dimensions, $C=C^{*}$. This Hamiltonian belongs to the BDI class. The classification table [1-3] displays integer $\mathbb{Z}$, a topological index for $1 \mathrm{D}$ (Kane and Lubensky's chain [10]), and integer $\mathbb{Z}_{2}$, a topological index for $0 \mathrm{D}$.

In this paper we provide a zero-dimensional topological invariant and we interpret the meaning of the topological transition in quantum dots and mechanical systems in the BDI class. On the phononic side, a zero-dimensional Maxwell lattice would imply that there are no degrees of freedom (dof), providing the usual equation used in the literature: $N_{\text {dof }}=$ $d N_{\text {sites }}$. However, we define a zero-dimensional Maxwell lattice as a mechanical lattice with a finite number of degrees of freedom. We design Maxwell lattices with 1 degree of freedom per site, but this is not necessarily the case when defining a zero-dimensional mechanical system. We choose to focus on two specific Maxwell lattices in zero dimensions with $N_{\text {dof }}=2$ and 3 , and we complete the table of mechanical topological insulators. The rest of this paper is organized as follows. In Sec. II we compute the topological index for a general BDI Hamiltonian together with a physical interpretation. In Sec. III we study two illustrative examples for electronic quantum dots, and in Sec IV we design gapped materials in zero dimensions with different topological indexes and translate previous interpretation's findings into these elastic and isostatic systems. 


\section{ZERO-DIMENSIONAL TOPOLOGICAL INVARIANT IN BDI CLASS}

The computation of the zero-dimensional topological index is explained below.

Method 1. From a Hamiltonian with chiral symmetry we define the matrix $C$ as the block off-diagonal part [the compatibility matrix in mechanical systems as in Eq. (1)]. The procedure to determine if two Hamiltonians, $H_{1}$ and $H_{2}$, belong to different topological classes consists of constructing an interpolation between $C_{1}$ and $C_{2}$, the limits being $\tilde{C}(t=0)=C_{1}$ and $\tilde{C}(t=\pi)=C_{2}$. An interpolation such as $\tilde{C}(t)$ must fulfill the constraints imposed by the class BDI:

$$
\tilde{C}(t)=\tilde{C}^{*}(-t) \text {. }
$$

Now we upgrade the parameter $t$ to a momentum, $k$. As if we were dealing with a $1 \mathrm{D}$ system in BDI $\tilde{C}(k)$, we compute the corresponding topological invariant,

$$
\nu_{1}=\frac{i}{2 \pi} \int_{B Z} \operatorname{Tr}\left(\tilde{C}(k)^{-1} \partial_{k} \tilde{C}(k)\right) d k,
$$

where $v_{1}$ is an integer and the topological invariant in zero dimensions is defined as

$$
v_{0}=(-1)^{v_{1}} .
$$

This method seems to contain certain ambiguities in the interpolation we chose. Two different interpolations can indeed give different winding numbers $v_{1}$. However, Ref. [22], where a similar computation is provided, includes a proof of $v_{1}$ that is well defined up to mod 2. Therefore, $v_{0}$ is independent of the interpolation. In order to have an absolute index, we can set $C_{1}$ to a fixed matrix (the identity matrix) and determine if $C_{2}$ is trivial or not with respect to the identity. Notice the identity matrix will have $v_{0}=1$.

As we mention above, a similar version for this computation is given in Ref. [22] for electronic systems. Instead of working with the $C$ matrix, Ryu et al. [22] define $q$ as the block offdiagonal part of the spectral projection onto the filled Bloch states:

$$
R=1-2 \sum_{i}^{N_{\text {filled }}}\left|u_{i}\right\rangle\left\langle u_{i}\right|=\left(\begin{array}{cc}
0 & q \\
q^{\dagger} & 0
\end{array}\right) .
$$

Both methods are consistent and give the same result. We keep the compatibility matrix representation, due to its meaning in mechanical systems.

Method 2. We define a physical property equivalent to the above-defined invariant $v_{0}$. We relate the Hamiltonian to the sublattice symmetry of its occupied levels. We find that the relative phase between sublattices within eigenvectors is what changes at the topological transition.

By construction $H_{C}$ [Eq. (1)] is chiral symmetric, $P H_{C}=-H_{C} P$, where the symmetry operator in the basis in which the Hamiltonian in block off-diagonal reads $P=\sigma_{z} \otimes I$. (We work here with isostatic systems, or in other words with square off-diagonal blocks.) This intrinsic symmetry guarantees levels come in pairs $\pm E$ with eigenvectors,

$$
\left|\psi_{ \pm}^{(j)}\right\rangle=\left(\begin{array}{c}
\psi_{a}^{(j)} \\
\pm \psi_{b}^{(j)}
\end{array}\right)
$$

where $a$ and $b$ label the two sublattices and $j=\left\{1, \ldots, N_{C}\right\}$ enumerates the levels ( $N_{C}$ is the dimension of the $C$ matrix).

For illustrative purposes, let us assume the off-diagonal part of the Hamiltonian is symmetric, $C=C^{t}$, and therefore is diagonalizable in real space, with nondegenerate eigenvalues. Then, the Hamiltonian and eigenvectors can be written as $H=\sigma_{x} \otimes q$ and

$$
\left|\psi_{ \pm}^{(j)}\right\rangle=\left(\begin{array}{c}
\psi_{C}^{(j)} \\
\pm \psi_{C}^{(j)}
\end{array}\right)
$$

respectively, with the corresponding energies $\pm E_{C}^{(j)}$. The subindex $C$ means they are the eigenvalues and eigenvectors of the $C$ matrix. The superindex $j$ enumerates $C$-matrix eigenvalues. For symmetric $C, \psi_{a}^{(j)}= \pm \psi_{b}^{(j)}$ for each $\left|\psi_{ \pm}^{(j)}\right\rangle$.

At the level crossing, the last occupied level $j=N_{C}$ changes the sign of energy $E_{C}^{\left(N_{C}\right)}$, and the occupied level will be, after the transition, the one with the opposite sublattice phase. Unlike other zero-dimensional Hamiltonians, topological transitions in the BDI class do not require an open system.

In order to keep track of such phase changes in the last occupied level we define $n_{N_{C}}$ for the last occupied band near the transition as

$$
n_{N_{C}}=2\left\langle\psi_{C}^{\left(N_{C}\right)} \mid \pm \psi_{C}^{\left(N_{C}\right)}\right\rangle= \pm 1,
$$

which changes sign at the transition. The factor of 2 is needed for normalization. Notice that two successive changes of the sign return the invariant to the initial value and thus the system to the same topological phase. Therefore, there are only two different phases $\left(\mathbb{Z}_{2}\right)$. Away from the transition, there may be other crossings at nonzero energy between levels, where the last occupied band can also change its sublattice phase.

In order to avoid counting those phase changes and extrapolating this result for nonsymmetric $C$ matrices, we need to define an index that keeps track of the transitions. In general $\psi_{a}$ and $\psi_{b}$ are independent, and

$$
n_{j}=\left\langle\psi_{a}^{j} \mid \pm \psi_{b}^{j}\right\rangle
$$

is a continuous quantity and no longer restricted to two values. $n_{j}$ will have divergent derivatives at the transitions, when $\psi_{b}^{j} \rightarrow-\psi_{b}^{j}$.

We work with the single-value decomposition of the compatibility matrix, $C=U \Sigma V^{t}$. Since $C$ is a real matrix, $U$ and $V$ are orthonormal matrices and their product, $U V^{t}$, is uniquely defined for nondegenerate single values, $\Sigma$. The subgroup of orthogonal matrices with the determinant +1 is disconnected from the set of the determinant -1 . Because at the transition a permutation of two eigenvectors occurs $\left(\left|\psi_{ \pm}\right\rangle \rightarrow\left|\psi_{\mp}\right\rangle\right)$, the determinant of $U V^{t}$ captures the essence of the topological transition in zero dimensions for an arbitrary compatibility matrix. Notice the index will be 1 when $C$ is the identity matrix. Information about all energy levels is contained in the orthogonal matrix $U V^{t}$. For AIII Hamiltonians, the reality condition of the off-diagonal blocks is not necessary; the analog $U V^{\dagger}$ is a unitary matrix with continuous values of its determinant.

Moreover, $n_{0}=\operatorname{det}\left(U V^{t}\right)$ is well defined in each topological phase and only changes at the topological transition as computed with Eqs. (4) and (5), $n_{0}=v_{0}$. 
1

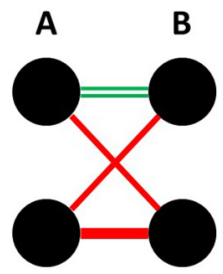

1

2

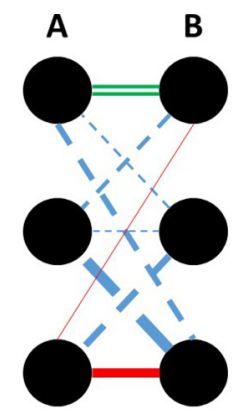

FIG. 1. (Left) Quantum dot model in the BDI topological class with two sites and two sublattices. The $C$ matrix is $2 \times 2$. (Right) Quantum dot model in the BDI topological class with three sites and two sublattices. The $C$ matrix is $3 \times 3$. The hopping intensity is represented by line width. The sign is positive for solid lines and negative for dashed lines. A double line represents the varying parameters in our examples. A and B label the sublattices.

\section{ELECTRONIC QUANTUM DOT}

We test computation methods of the topological invariant with electronic systems. We construct Hamiltonians with chiral symmetry in the BDI class, vary a hopping parameter, and study the topological phases. The Hamiltonians can be engineered into an electronic quantum dot with appropriate hoppings. We save the mechanical interpretation for models based on real elastic constraints.

\section{A. Example: Symmetric $C$}

Let us take a $2 \times 2 C$ matrix,

$$
H_{C}=\left(\begin{array}{llll}
0 & 0 & a & b \\
0 & 0 & b & c \\
a & b & 0 & 0 \\
b & c & 0 & 0
\end{array}\right),
$$

with 0.5 and $c=1$. We study the system for several values of $a$. Here $a$ is the hopping between site 1 from lattice A (B) to lattice $\mathrm{B}(\mathrm{A})$, and it is treated as the driving parameter. A simple cartoon of this model is depicted in Fig. 1 (left-hand panel). The spectrum is shown in Fig. 2. We compute the topological index for each value of $a$ with both methods described above. In Method 1, we use the interpolation

$$
\tilde{C}(k)=\frac{1}{2}(C+I)+e^{i k} \frac{1}{2}(C-I) .
$$

Notice it fulfills the limit conditions for $k=0$ and $k=\pi$, and it also satisfies $C(k)=C^{*}(-k)$.

Method 2 provides a major insight. Given the eigendecomposition of the $C$ matrix,

$$
E_{C}^{\mp}=\frac{1}{2}\left(a+c \mp \sqrt{(a-c)^{2}+4 b^{2}}\right),
$$

and $U_{C}$, the Hamiltonian $H_{C}$ eigendecomposition is $E_{H}=$ $\pm E_{C}^{\mp}$, and

$$
U_{H}=\frac{1}{\sqrt{2}}\left(\begin{array}{cc}
1 & 1 \\
-1 & 1
\end{array}\right) \otimes U_{C}=\frac{1}{\sqrt{2}}\left(\begin{array}{cc}
U_{C} & U_{C} \\
-U_{C} & U_{C}
\end{array}\right) .
$$

Here $U_{H}$ columns from 1 to $N_{C}$ are the eigenvectors corresponding to $-E_{C}^{\mp}$ and its sublattice symmetry is $\left|\psi_{-}\right\rangle$[Eq. (8)], while columns from $N_{C}+1$ to $2 N_{C}$ correspond to $E_{C}^{\mp}$ and

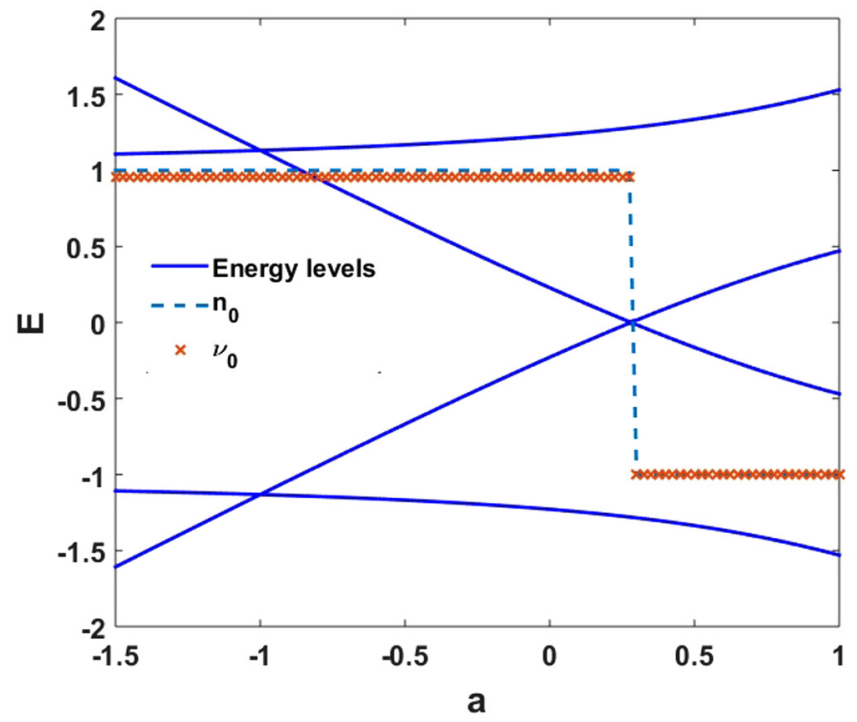

FIG. 2. Solid lines represent the four energy levels of the zerodimensional quantum dot with symmetric $C$ as a function of $a$. The " $x$ " symbols represent the computation of the topological index with Method 1. The dashed line represents the computation of the topological index with Method 2.

its sublattice symmetry is $\left|\psi_{+}\right\rangle$[Eq. (8)]. At the transition two eigenvalues switch sign and the occupied level changes discontinuously from $\left|\psi_{ \pm}\right\rangle$to $\left|\psi_{\mp}\right\rangle$. The sublattice symmetry protects the level crossing and the last occupied level changes sublattice symmetry as explained in Sec. II. The permutation of two columns change the sign of the determinant of $U_{H}$. Below, we see how $U_{C}$ relates with the rotation matrices of the single-value decomposition.

For $a=1$, both eigenvalues are positive and, therefore, equal to the single values. The single-value rotation matrices $U$ and $V$ are the same as the matrices of the eigenvectors. In that case, $U V^{t}=I$ and the Hamiltonian $H_{C}$ has the same topology as the identity matrix. As the value of $a$ is decreased, $E_{C}^{-}$changes sign; by definition the single values are always positive and this difference is captured by the rotation matrices. Namely, $\operatorname{det}\left(U V^{t}\right)=-1$ if the signs of the two $C$-matrix eigenvalues are different.

Results from the two methods are shown in Fig. 2. In the range of parameters explored, $E_{C}^{-}=0$ changes sign for $a_{c}=$ $\frac{b^{2}}{c}=0.25$. Both methods agree. For $a>a_{c}$, the Hamiltonian $\stackrel{c}{H}_{C}$ is topologically equivalent to the identity matrix and $n_{0}=$ $v_{0}=1$, whereas for $a<a_{c}$ the Hamiltonian is nonequivalent and $n_{0}=v_{0}=-1$.

\section{B. Example: Nonsymmetric $C$}

We use a $3 \times 3$ random matrix whose hoppings are depicted in Fig. 1 (right-hand panel). We vary the first term of the diagonal (hopping between site 1 in the two sublattices) and study the topological phases. Crossings at zero energy signal a topological transition. Results of the energy levels and topological index are shown in Fig. 3. Notice that avoiding crossings occurs at $a=0.52$, the two noncrossing levels are not protected by any symmetry. In Fig. 4, we plot the determinant of $\tilde{C}(k)$ according to Method 1 and using the interpolation of 


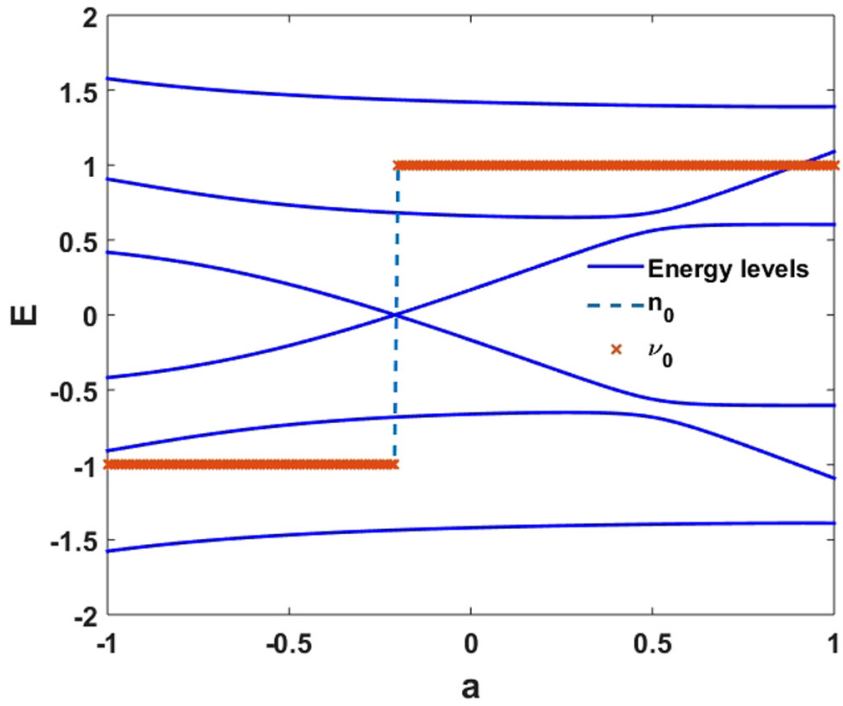

FIG. 3. Solid lines represent the four energy levels of the zerodimensional quantum dot with a nonsymmetric $C$ matrix as a function of $a$. The "x" symbols represent the computation of the topological index with Method 1. The dashed line represents the computation of the topological index with Method 2.

Eq. (12). An "x" symbol signals $\tilde{C}(k=0)=C$; also $\tilde{C}(k=$ $\pm \pi)=I=1$.

\section{MECHANICAL CASE}

In this section we propose a mechanical topological insulator in OD and study the meaning of its phases. Unlike electronic systems in which nonzero hoppings might appear within the same sublattice, chiral symmetry in mechanical systems is exact by definition [see Eq. (1)].

\section{A. Model}

We propose a $3 \times 3$ compatibility matrix, therefore we have three constraints and three degrees of freedom dof. The model is depicted in Fig. 5. It consists of three masses constrained to move in a single direction (along the tubes in which each one is contained). Each mass is attached to the other degrees of

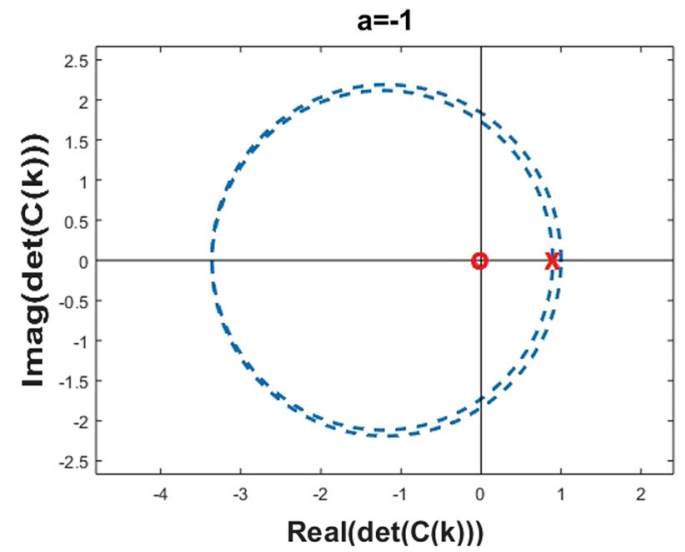

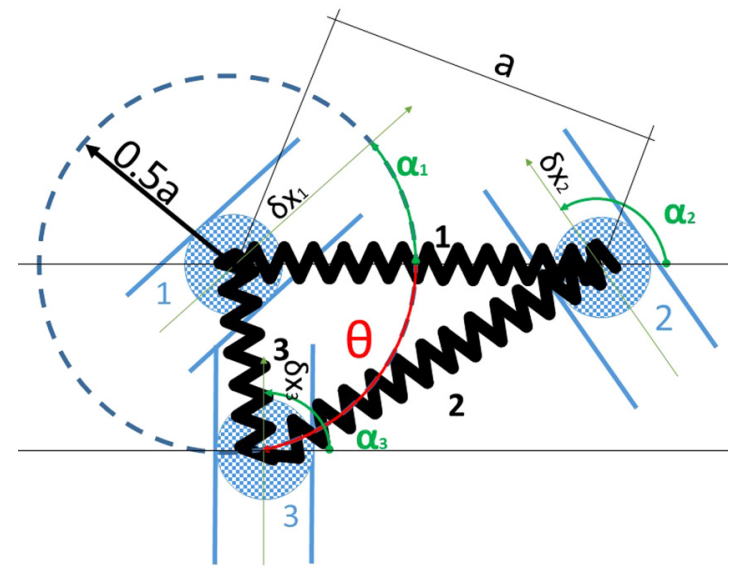

FIG. 5. Mechanical model in zero dimensions. The three masses are represented by circles shaded with a checkerboard pattern and are constrained to move in a single direction. Equilibrium is determined by $a, \alpha_{1}, \alpha_{2}, \alpha_{3}$, and $\theta$.

freedom by a spring; all of them have the same elastic constant. The degrees of freedom are $\delta x$, positive in the direction of the corresponding arrow. The equilibrium is defined by the angle of spring 3 respect to the angle of spring 1, $\theta$, and the distance $a$ (see Fig. 5). The equilibrium positions are

$$
\left\{\begin{array}{l}
\vec{R}_{1}=(0,0), \\
\vec{R}_{2}=(a, 0), \\
\vec{R}_{3}=\frac{a}{2}[\cos (\theta), \sin (\theta)],
\end{array}\right.
$$

and the displacements are

$$
\vec{\delta}_{x i}=\delta_{x i}\left[\cos \left(\alpha_{i}\right), \sin \left(\alpha_{i}\right)\right],
$$

where $\alpha_{1}=\frac{\pi}{3}, \alpha_{2}=\frac{2 \pi}{3}$, and $\alpha_{3}=\frac{\pi}{2}$. The bond directions are $\vec{b}_{i}=\left(\vec{R}_{i+1}-\vec{R}_{i}\right) / l_{i}$, with $l_{i}=\left|\vec{R}_{i+1}-\vec{R}_{i}\right|$ and $\vec{R}_{4}=\vec{R}_{1}$.

We can write the compatibility matrix as

$$
C=\left(\begin{array}{ccc}
\vec{u}_{1} \cdot \vec{b}_{1} & -\vec{u}_{2} \cdot \vec{b}_{1} & 0 \\
0 & \vec{u}_{2} \cdot \vec{b}_{2} & -\vec{u}_{3} \cdot \vec{b}_{2} \\
-\vec{u}_{1} \cdot \vec{b}_{3} & 0 & \vec{u}_{3} \cdot \vec{b}_{3}
\end{array}\right),
$$

where $\vec{u}_{i}=\frac{\partial \vec{\delta}_{x i}}{\partial \delta_{x i}}$.

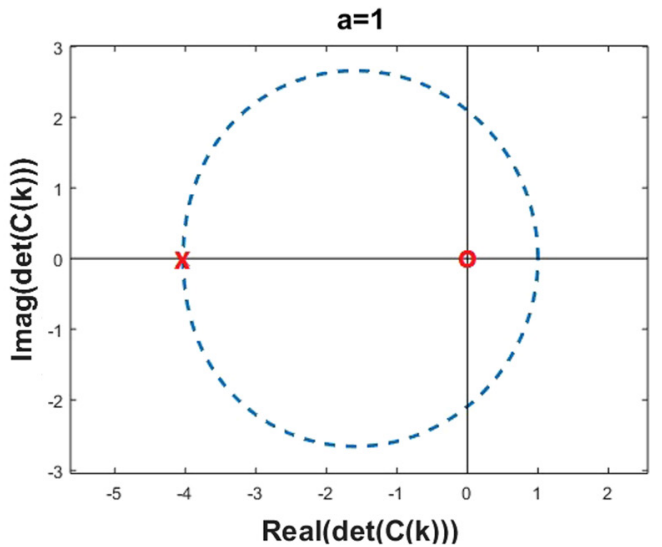

FIG. 4. Winding of determinant $\tilde{C}(k)$ as described by Method 1, for different values of $a$ : (right-hand panel) $a=-1$ and (Left-hand panel) $a=1$. The "x" symbol signals $k=0$. The "o" symbol represents the origin. 


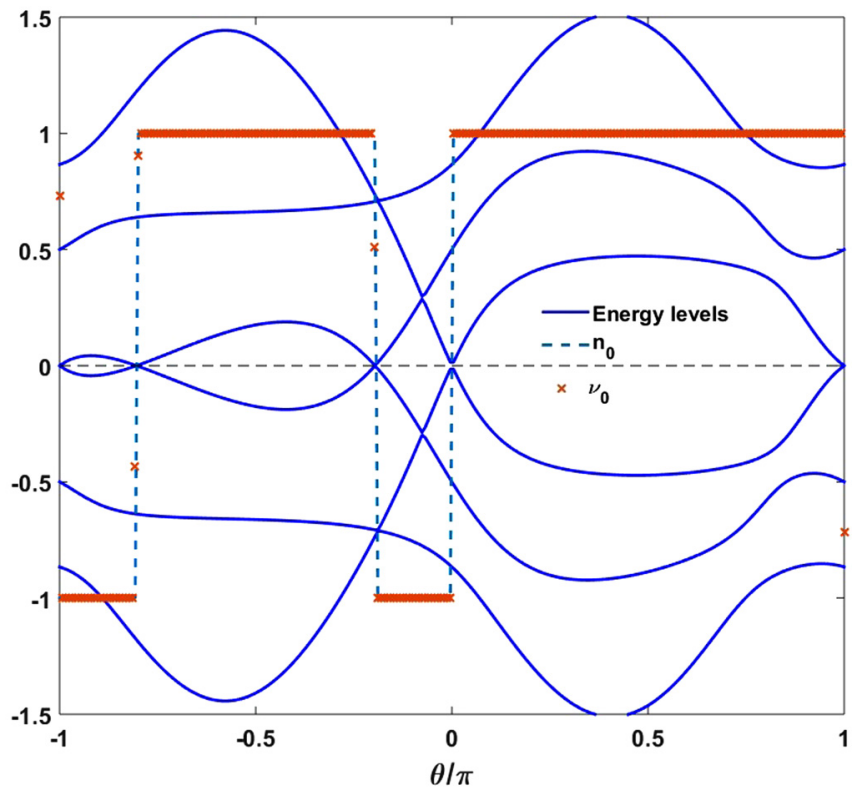

FIG. 6. Energy levels of the mechanical Maxwell lattice in zero dimensions as a function of $\theta$. The " $x$ " symbols denote the computation of the topological index with Method 1. The dashed dashed line denotes the computation of the topological index with Method 2.

In Fig. 6 the energy levels of the mechanical system are shown [the negative energies arise from the Hamiltonian construction (1)]. We compute the topological index of two zero-dimensional Hamiltonians. Using the interpolation function Eq. (12) (method 1) and also Method 2, the two possible topological phases appear. Transitions occur at $\theta=\{-0.8 \pi,-0.2 \pi, 0, \pi\}$ as can be seen in Fig. 6 .

\section{B. Interpretation}

The two sublattices in the mechanical case represent different quantities: positions and tensions or forces and elongations. The sign of positions and forces specifies the direction of displacement with respect to the equilibrium position, the sign of elongation specifies extension or contraction, and the sign of tension determines whether the spring pulls or pushes a mass. They are all interconnected, and the information in the Hamiltonian, by construction, is duplicated. A different sublattice phase informs us whether a displacement along a normal mode will cause a combination of elongations, forces, and tensions in one sense or the opposite.

In the following, elongations, forces, tensions, and displacement will be represented by $e, f, t$, and $x$, respectively. All elongations of the system are described in a column vector, $e=\left(e_{1}, e_{2}, e_{3}\right)^{t}$. The enumeration is described in Fig. 5, spring enumeration is used for elongations and tensions, and mass enumeration is used for positions and forces.

The energies are given in pairs, so let us write the two equations extracted from the diagonalized version of the Hamiltonian. First,

$$
\left(\begin{array}{c}
e \\
-f
\end{array}\right)=H_{C}\left(\begin{array}{l}
t \\
x
\end{array}\right)
$$

and after diagonalization

$$
U_{H}^{t}\left(\begin{array}{c}
e \\
-f
\end{array}\right)=\hat{E} U_{H}^{t}\left(\begin{array}{l}
t \\
x
\end{array}\right),
$$

where $\hat{E}$ is a diagonal matrix with the diagonal $\left(-\left|E_{3}\right|,-\left|E_{2}\right|\right.$, $\left.-\left|E_{1}\right|,\left|E_{1}\right|,\left|E_{2}\right|,\left|E_{3}\right|\right)$ and $\left|E_{1}\right|<\left|E_{2}\right|<\left|E_{3}\right|$. Writing the equation for a pair of opposite energies, $\pm E_{j}$, we get

$$
\begin{aligned}
\psi_{a}^{(j)} e & =E_{j} \psi_{b}^{(j)} x, \\
\psi_{b}^{(j)} f & =-E_{j} \psi_{a}^{(j)} t,
\end{aligned}
$$

where $\left[\left(\psi_{a}^{(j)}\right)^{t}, \pm\left(\psi_{b}^{(j)}\right)^{t}\right]^{t}$ is the column of $U_{H}$ corresponding to the eigenvalues $\pm E_{j}$. Notice that in the previous equation we lose the information about the relative phase between $\psi_{a}^{(j)}$ and $\psi_{b}^{(j)}$ because the sign will change both in $\psi_{b}^{(j)}$ and energy $E_{j}$.

We can prove these equations. First, if we move the masses according to $\psi_{b}^{(j)}$,

$$
\vec{X}_{i}=\vec{R}_{i}+\epsilon \vec{u}_{i} \psi_{b}^{(j)}(i),
$$

where $\epsilon$ is a small positive number compatible with a linear regime, and we excite a mode with the energy $+\left|E_{j}\right|$. The elongations caused by this displacement are $e_{i} \sim \psi_{a}^{j}(i)$ and not the opposite. The proportional factor is $+\left|E_{j}\right|$. At the transitions, the elongation relative phase changes abruptly.

Second, if we apply a tension to bonds according to $\psi_{a}^{(j)}$,

$$
\vec{t}_{i}=\epsilon \psi_{a}^{(j)}(i) \vec{b}_{i}
$$

we produce a force in each mass of

$$
f_{i}=\left(t_{i} \vec{b}_{i}-t_{i-1} \vec{b}_{i-1}\right) \cdot \vec{u}_{i},
$$

which is proportional to $f_{i} \sim-\psi_{b}^{(j)}(i)$ and not the opposite. The proportional factor is $+\left|E_{j}\right|$.

We need positive and negative energy equations in order to solve the system. Therefore, they are not different states (occupied and empty) as in electronics. With the dynamical matrix we will only know $\psi_{b}^{(j)}$, and with its dual partner we will extract $\psi_{a}^{(j)}$ as well. We could also check the equations of motion with the Lagrangian approach:

$$
L=\sum_{i} \frac{1}{2} m \dot{x}_{i}^{2}-\frac{1}{2} \kappa\left(x_{i}-x_{i+1}\right)^{2} .
$$

From Euler-Lagrange equations we can derive a matrix relating forces with displacement (Hook's law):

$$
f=\left(\begin{array}{ccc}
-2 & \vec{u}_{1} \cdot \vec{u}_{2} & \vec{u}_{1} \cdot \vec{u}_{3} \\
\vec{u}_{2} \cdot \vec{u}_{1} & -2 & \vec{u}_{2} \cdot \vec{u}_{3} \\
\vec{u}_{3} \cdot \vec{u}_{1} & \vec{u}_{3} \cdot \vec{u}_{2} & -2
\end{array}\right) x .
$$

If $x=\psi_{b}^{(j)}, f \sim-\psi_{b}^{(j)}$, as expected.

Now, we make connection with topological mechanics literature. In higher dimensions topological zero-energy edge states appear as a consequence of the $\mathbb{Z}$ invariant in $1 D$. The zero modes and its dual partners, the states of self-stress, are of recent interest. From a fundamental point of view, they are the main signature of topological mechanics. In applications, they control the response to stress [17], can contribute to the control of fracture [23], and are used to design mechanical 


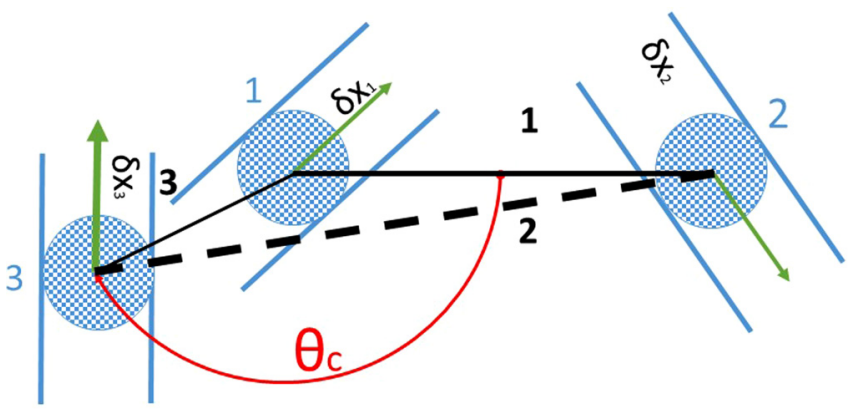

FIG. 7. Zero eigenvalues of $H_{C}$ at $\theta_{c}=-0.8 \pi$. The zero mode is indicated by arrows (length and width are proportional to the normal mode displacement) and the state of self-stress is indicated by solid $(+)$ and dashed $(-)$ thickened lines.

diodes. In our model when a phononic energy level goes to zero, because energy levels in $H_{C}$ appear in pairs, $\pm E$, they are degenerate and the two eigenvectors can be written with nonzero terms in one of the sublattices only: $\left|\psi_{S S S}\right\rangle=\left(\phi_{a}, 0\right)^{t}$ and $\left|\psi_{0}\right\rangle=\left(0, \phi_{b}\right)^{t}$. In Maxwell lattices, these modes are called states of self-stress (SSS) and zero or floppy modes, respectively $[10,11]$. The eigenvector $\psi_{0}$ means that a certain combination of displacements does not cause the elongation of any spring, and therefore there is no elastic energy. $\psi_{S S S}$ means that a combination of tensions in springs does not cause a net force (see Fig. 7). We can express these two eigenvectors in the symmetric and antisymmetric bases [Eq. (7)]:

$$
\psi_{ \pm}=\frac{1}{\sqrt{2}}\left(\psi_{0} \pm \psi_{S S S}\right),
$$

where a combination of displacement and elongations will be the mode of the mechanical Hamiltonian that acquires energy away from the transition and the same combination of tensions will cause the opposite combination of forces as explained above. It is worth mentioning that the dynamical matrix $D=C^{t} C$ only contains information on the normal modes, or displacements $\psi_{b}$, and its dual partner $\tilde{D}=C C^{t}$ only contains information on "normal tensions," $\psi_{a}$. But it is $H_{C}$ which contains the relative phase between the two, the topological information.

\section{v. CONCLUSIONS}

In this paper we design a zero-dimensional mechanical insulator at the verge of mechanical instability with nontrivial topological properties. Isostatic frames can be studied with a Hamiltonian construction based on the square root of the dynamical matrix $D$ and its superpartner $\tilde{D}$ [10]. The BDI class in real space dimensions only has a topological invariant in zero dimensions and one dimension. Being the last one, the 1D-chain analog to SSH [10] together with extensions to higher dimensions, the most studied. We complete the table with a feasible example in zero dimensions and explain the meaning of the topological invariant in both electronic and mechanical systems. BDI Hamiltonians have energies in pairs, $\pm E$. Due to their different sublattice symmetries, level crossing at zero energy is allowed, according to von Neumann-Wigner theorem of quantum mechanics. At the zero-energy level crossing, quantum dots' occupied states change the sublattice symmetry, signaling a topological transition. In mechanical frameworks, the two sublattices correspond to different physical quantities (mass displacement and bond elongation or bond tension and mass net force) and their interplay gives rise to different topological phases.

\section{ACKNOWLEDGMENTS}

N.L. thanks Shinsei Ryu and Kai Sun for fruitful discussions and insights. N.L. acknowledges financial support from the Spanish Ministry of Economy and Competitiveness, through The María de Maeztu Programme for Units of Excellence in R\&D (Grant No. MDM-2014-0377). This work has been funded by MINECO Grant No. FIS2015-64886-C5-5-P.
[1] A. Kitaev, Periodic table for topological insulators and superconductors, AIP Conf. Proc. 1134, 22 (2009).

[2] A. P. Schnyder, S. Ryu, A. Furusaki, and A. W. W. Ludwig, Classification of topological insulators and superconductors in three spatial dimensions, Phys. Rev. B 78, 195125 (2008).

[3] A. P. Schnyder, S. Ryu, A. Furusaki, and A. W. W. Ludwig, Classification of topological insulators and superconductors, AIP Conf. Proc. 1134, 10 (2009).

[4] C. W. J. Beenakker, J. M. Edge, J. P. Dahlhaus, D. I. Pikulin, S. $\mathrm{Mi}$, and M. Wimmer, Wigner-Poisson Statistics of Topological Transitions in a Josephson Junction, Phys. Rev. Lett. 111, 037001 (2013).

[5] A. Sakurai, Comments on superconductors with magnetic impurities, Prog. Theor. Phys. 44, 1472 (1970).

[6] A. Y. Kitaev, Unpaired Majorana fermions in quantum wires, Phys.-Usp. 44, 131 (2001).

[7] A. V. Balatsky, I. Vekhter, and J.-X. Zhu, Impurity-induced states in conventional and unconventional superconductors, Rev. Mod. Phys. 78, 373 (2006).
[8] C. P. Broedersz, X. Mao, T. C. Lubensky, and F. C. MacKintosh, Criticality and isostaticity in fibre networks, Nat. Phys. 7, 983 (2011).

[9] A. J. Liu and S. R. Nagel, Jamming is not just cool any more, Nature (London) 396, 21 (1998).

[10] C. L. Kane and T. C. Lubensky, Topological boundary modes in isostatic lattices, Nat. Phys. 10, 39 (2014).

[11] T. C. Lubensky, C. L. Kane, X. Mao, A. Souslov, and K. Sun, Phonons and elasticity in critically coordinated lattices, Rep. Prog. Phys. 78, 073901 (2015).

[12] D. Z. Rocklin, B. G.-g. Chen, M. Falk, V. Vitelli, and T. C. Lubensky, Mechanical Weyl Modes in Topological Maxwell Lattices, Phys. Rev. Lett. 116, 135503 (2016).

[13] O. Stenull, C. L. Kane, and T. C. Lubensky, Topological Phonons and Weyl Lines in Three Dimensions, Phys. Rev. Lett. 117, 068001 (2016).

[14] J. Paulose, B. G.-g. Chen, and V. Vitelli, Topological modes bound to dislocations in mechanical metamaterials, Nat. Phys. 11, 153 (2015). 
[15] H. C. Po, Y. Bahri, and A. Vishwanath, Phonon analog of topological nodal semimetals, Phys. Rev. B 93, 205158 (2016).

[16] D. Z. Rocklin, S. Zhou, K. Sun, and X. Mao, Transformable topological mechanical metamaterials, Nat. Commun. 8, 14201 (2017).

[17] D. Z. Rocklin, Directional mechanical response in the bulk of topological metamaterials, New J. Phys. 19, 065004 (2017).

[18] J. Paulose, A. S. Meeussen, and V. Vitelli, Selective buckling via states of self-stress in topological metamaterials, Proc. Natl. Acad. Sci. USA 112, 7639 (2015).
[19] B. G.-g. Chen, N. Upadhyaya, and V. Vitelli, Nonlinear conduction via solitons in a topological mechanical insulator, Proc. Natl. Acad. Sci. USA 111, 13004 (2014).

[20] G. Baardink, A. Souslov, J. Paulose, and V. Vitelli, Localizing softness and stress along loops in 3D topological metamaterials, Proc. Natl. Acad. Sci. USA 115, 489 (2018).

[21] N. Lera, J. V. Alvarez, and K. Sun (unpublished).

[22] S. Ryu, A. P. Schnyder, A. Furusaki, and A. W. W. Ludwig, Topological insulators and superconductors: Tenfold way and dimensional hierarchy, New J. Phys. 12, 065010 (2010).

[23] L. Zhang and X. Mao (unpublished). 